\title{
Aberrant functional connectivity of the cingulate subregions in right-sided temporal lobe epilepsy
}

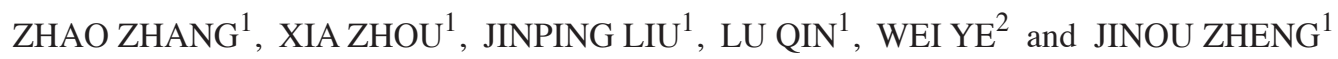 \\ Departments of ${ }^{1}$ Neurology and ${ }^{2}$ Radiology, The First Affiliated Hospital of Guangxi Medical University, \\ Nanning, Guangxi 530021, P.R. China
}

Received July 17, 2019; Accepted December 9, 2019

DOI: $10.3892 /$ etm.2020.8551

\begin{abstract}
Patients with temporal lobe epilepsy (TLE) have been indicated to exhibit abnormal resting-state functional connectivity (rsFC) of the cingulate cortex. However, it has remained elusive whether cingulate subregions show different connectivity patterns in TLE. The present study aimed to investigate the differences in rsFC of each cingulate subregion between patients with right-sided TLE (rTLE) and healthy controls (HCs), as well as their association with executive control performance in rTLE. A total of 27 patients with rTLE and 20 age-, sex- and education-matched healthy controls were recruited and all participants underwent resting-state functional MRI and an attention network test for the assessment executive control function. In each hemisphere, the cingulate gyrus (CG) was divided into CG-1 (dorsal area 23; A23d), CG-2 (rostroventral area 24; A24rv), CG-3 (pregenual area 32; A32p), CG-4 (ventral area 23; A23v), CG-5 (caudodorsal area 24; A24cd), CG-6 (caudal area 24; A23c) and CG-7 (subgenual area 32; A32sg). Pearson's correlation analysis was performed to assess the correlation between the altered FCs of the cingulate subregions and clinical variables. In patients with rTLE, the majority of the cingulate subregions exhibited decreased $\mathrm{rsFC}$; this was primarily restricted to the right CG-2, the bilateral CG-6 and the bilateral CG-7. Increased rsFC was only detected in rTLE restricted to the left CG-1. Impairments in executive control efficiency were identified in patients with rTLE in comparison with the HCs. Significant alterations in rsFC between the cingulate subregion and the brain regions were mostly decreased (and some slightly increased), suggesting that FC may potentially have a left-side advantage in patients with rTLE. FC variations of the
\end{abstract}

Correspondence to: Professor Jinou Zheng, Department of Neurology, The First Affiliated Hospital of Guangxi Medical University, 6 Shuangyong Road, Nanning, Guangxi 530021, P.R. China

E-mail: jinouzheng@163.com

Key words: temporal lobe epilepsy, cingulate cortex, subregion, resting-state functional magnetic resonance, attention network test, executive control cingulate subregions were indicated to be specific to rTLE. In addition, increased connectivity in the left CG-1 and left superior frontal gyrus were negatively correlated with executive control performance, suggesting a compensatory mechanism on executive control deficits in pathological conditions. This information on differentially altered FC patterns of the cingulate subregions may provide a deeper understanding of the complex neurological mechanisms and executive control dysfunctions underlying rTLE.

\section{Introduction}

Epilepsy is a neurological disorder which affects $>50$ million individuals worldwide (1). Temporal lobe epilepsy (TLE) is one of the most common types of partial epilepsy in adults, in which the epileptogenic foci are generally located in the temporal lobe (2). TLE is associated with various cognitive impairments such as attention deficit and memory impairment, and impacts patient quality of life. Recurrent abnormal neuronal discharges accelerate the risk of abnormal lesions in a number of brain regions, including the temporal and extratemporal regions, which may contribute to their functional impairment (3). However, the underlying pathophysiological and neurological mechanisms of TLE have remained elusive.

Previous studies on TLE have identified abnormal functional connectivity (FC) and structural abnormalities in specific brain regions, including the cingulate cortex (4-6). The cingulate cortex is a complex brain structure encompassing diverse functional domains (7). With a deeper understanding of TLE, diverse connectivity and functional heterogeneity in brain subregions have been reported in numerous studies $(8,9)$, which indicates higher demand and new challenges for future research.

The deep cingulate cortex encircles the corpus callosum and is considered to be one of the most prominent features of the inner brain (10). The cingulate cortex is the core center of activity in TLE, and has a crucial role in cognitive, sensory, motor and emotional processes (11). In a previous study, the mean diffusivity of the left cingulate cortex and parahippocampal cortex in patients with TLE was indicated to be significantly higher than that in the healthy control (HC) group (12). Another study revealed that in the TLE group, patients with unilateral hippocampal sclerosis exhibited lower fractional anisotropy of the ipsilateral cingulum than of the contralateral cingulum, which 
was also lower than that in the control group (4). In addition to widespread hypometabolism in brain regions ipsilateral to the epileptogenic focus, hypometabolism in areas including the anterior cingulate gyrus (CG), the middle $\mathrm{CG}$ and the thalamus were also observed on the contralateral epileptogenic foci of the TLE group as compared to the HCs (13). A functional MRI (fMRI) study revealed that decreased regional homogeneity was present primarily in the default mode network (DMN; including the precuneus, the posterior $\mathrm{CG}$ and the mesial prefrontal cortex) in patients with TLE, relative to the HC subjects (14). In another fMRI study, functional network abnormalities were identified in the limbic systems of patients with TLE (including the ipsilateral ventral anterior cingulate cortex and the hypothalamus, the subiculum and the posterior cingulate cortex), which was significantly correlated with seizure frequency (3). In neuroimaging studies, functional and structural alterations are frequently observed in the cingulate cortex of patients with TLE. These changes have been associated with TLE seizure frequency, disease duration (15) and attention deficits (including executive control of attention) (5). The various regions of the cingulate cortex are widely connected with different brain regions, and each have different functions (10). Hence, it was speculated that the cingulate cortex has a key role in TLE. Although previous studies have reported alterations in structural and functional connectivity of the cingulate cortex in TLE, it remains elusive whether the cingulate subregions exhibit differential intrinsic functional patterns in TLE. It should be noted that the cingulate cortex is comprised of separate subregions, and that there is a lack of a systematical investigation into the differences in functional connectivity of these subregions in TLE. The subregional specialization of the cingulate cortex is considered to be linked to structural and functional heterogeneity, as well as cytoarchitecture (16); Vogt et al (17) proposed that the cingulate cortex may be divided into four subregions based on integrated neurobiological assessments, which include the anterior cingulate cortex (ACC), the posterior cingulate cortex (PCC), the middle cingulate cortex (MCC) and the retrosplenial cingulate cortex (RSCC). In a diffusion tractography study based on probabilistic connectivity, Beckmann et al (18) identified nine cingulate subregions and determined that the structural connectivity of different subregions overlapped. Inspired by previous studies, Fan et al (19) proposed the Human Brainnetome Atlas, which is based on connectional architecture; the atlas divides the cingulate cortex into seven subregions, with a total of 14 sub-regions in the bilateral cingulate cortex. Given the differentiation of the cingulate subregions and their role in processes including conflict monitoring, response selection and memory, this may provide a foundation for further studies of the cingulate subregions in TLE.

Based on the aforementioned studies, the functional and structural alterations of the cingulate subregions are considered to be closely associated with TLE. The purpose of the present study was to investigate specific changes in the FC patterns of the cingulate cortex (at the subregional level) in patients with right-sided TLE (rTLE), using resting-state fMRI (rs-fMRI). Subsequently, the attention network test (ANT) (20) was used to determine the executive control performance of each participant. The potential association between abnormal cingulate subregion connectivity and clinical characteristics was also investigated.

\section{Materials and methods}

\section{Study participants}

rTLE group. A total of 27 consecutively patients with rTLE

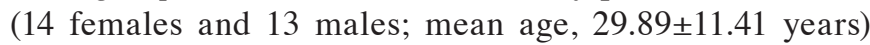
were recruited between July 2015 and January 2018 at the Epilepsy Clinic of the First Affiliated Hospital of Guangxi Medical University (Nanning, China). The present study was approved by the Medical Research Ethics Committee of the First Affiliated Hospital of Guangxi Medical University and written informed consent was obtained from each participant. The subjects were recruited based on standard and vide o-electroencephalographic (EEG) evaluation, brain MRI results and clinical data (including clinical characteristics, neurological examination results and medication regimens). Each patient exhibited typical temporal lobe seizures and was diagnosed with TLE based on the criteria and therapeutic guidelines of the International League Against Epilepsy (21). The diagnosis was based on clinical characteristics, confirmative video-EEG monitoring and imaging examinations, and was determined by two experienced neurologists. Patients with rTLE met at least two of the following inclusion criteria: i) The clinical symptoms of TLE indicated epileptic lesions in the temporal lobe; ii) MRI results suggested atrophy, sclerosis localized to the right hippocampus or other abnormalities in the right temporal lobe; and iii) EEG abnormalities revealed ictal or interictal discharges originating from the right temporal lobe. The exclusion criteria were as follows: i) A Mini-Mental State Examination (MMSE) score of <24; ii) any history of severe neurological or mental diseases; iii) traumatic brain injury or other serious medical conditions.

HC group. The HC group included 20 healthy subjects without rTLE ( 8 females and 12 males; mean age, 27.70 \pm 2.79 years) who were age-, sex- and education-matched with 20 of those from the rTLE group. The HC group were recruited from the community between July 2015 and January 2018. In addition, the HCs had normal vision (or at least it was corrected to normal) and no history of neurological or psychiatric disorders. All participants recruited had an MMSE score of $>24$, which was also closely matched with that of the corresponding patients with rTLE. All participants were right-handed.

fMRI data acquisition. All rs-fMRI data were acquired using a 3-T Achieva MRI scanner (Philips Healthcare) with a 12-channel phased-array head coil, and tight clamps were used to limit head movement. Resting-state functional images were acquired using a gradient-echo echo-planar imaging (EPI) sequence with the following scanning parameters: Repetition time/echo time, $2,000 / 30 \mathrm{msec}$; flip angle, $90^{\circ}$; number of slices, 31; pitch, $1 \mathrm{~mm}$; field of view, 220x $220 \mathrm{~mm}$; matrix, $64 \times 64$; voxel size, $3.44 \times 3.44 \times 6.00 \mathrm{~mm}$; and 180 volumes. During scanning, the subjects were instructed to lie still, keeping their eyes closed but staying awake, and not to think of anything in particular.

fMRI data processing. The DPABI software in MATLAB ${ }^{\circledR}(22)$ was utilized for pre-processing the resting-state fMRI data, and the first 10 time-points were removed. Slice timing and head motion corrections were subsequently performed. 
Table I. Name and coordinates of the cingulate subregions in the Human Brainnetome Atlas.

\begin{tabular}{|c|c|c|c|c|}
\hline \multirow[b]{2}{*}{ Label ID/subregion, hemisphere } & \multirow[b]{2}{*}{ Abbreviation } & \multicolumn{3}{|c|}{ MNI coordinates } \\
\hline & & $\mathrm{X}$ & $\mathrm{Y}$ & $\mathrm{Z}$ \\
\hline \multicolumn{5}{|l|}{ CG-1/dorsal area 23} \\
\hline $\mathrm{L}$ & L-A23d & -4 & -39 & 31 \\
\hline $\mathrm{R}$ & R-A23d & 4 & -37 & 32 \\
\hline \multicolumn{5}{|l|}{ CG-2/ rostroventral area 24} \\
\hline $\mathrm{L}$ & L-A24rv & -3 & 8 & 25 \\
\hline $\mathrm{R}$ & $\mathrm{R}-\mathrm{A} 24 \mathrm{rv}$ & 5 & 22 & 12 \\
\hline \multicolumn{5}{|l|}{ CG-3/pregenual area 32} \\
\hline $\mathrm{L}$ & L-A32p & -6 & 34 & 21 \\
\hline $\mathrm{R}$ & R-A32p & 5 & 28 & 27 \\
\hline \multicolumn{5}{|l|}{ CG-4/ventral area 23} \\
\hline $\mathrm{L}$ & L-A23v & -8 & -47 & 10 \\
\hline $\mathrm{R}$ & R-A23v & 9 & -44 & 11 \\
\hline \multicolumn{5}{|l|}{ CG-5/caudodorsal area 24} \\
\hline $\mathrm{L}$ & L-A24cd & -5 & 7 & 37 \\
\hline $\mathrm{R}$ & $\mathrm{R}-\mathrm{A} 24 \mathrm{~cd}$ & 4 & 6 & 38 \\
\hline \multicolumn{5}{|l|}{ CG-6/caudal area 24} \\
\hline $\mathrm{L}$ & L-A23c & -7 & -23 & 41 \\
\hline $\mathrm{R}$ & $\mathrm{R}-\mathrm{A} 23 \mathrm{c}$ & 6 & -20 & 40 \\
\hline \multicolumn{5}{|l|}{ CG-7/subgenual area 32} \\
\hline $\mathrm{L}$ & L-A32sg & -4 & 39 & -2 \\
\hline $\mathrm{R}$ & R-A32sg & 5 & 41 & 6 \\
\hline
\end{tabular}

All coordinates are in association with the MNI template. X, Y and Z refer to coordinates of the primary peak locations in the MNI space. MNI, Montreal Neurological Institute; CG, cingulate gyrus; L, left; R, right.

Functional data were spatially normalized to the Montreal Neurological Institute EPI template, resampled to a resolution of $3 \times 3 \times 3 \mathrm{~mm}$, and smoothed with a $6-\mathrm{mm}$ Gaussian kernel; this was followed by bandpass filtering (range, 0.01-0.08 Hz). Sources of spurious variance, including averaging signals from white matter, cerebrospinal fluid, the global brain and the six head-motion parameters, were removed from the imaging data (7). Data from participants with head motion $>2 \mathrm{~mm}$ or head rotations $>2^{\circ}$ were excluded from further calculations.

Definition of cingulate gyrus (CG) subregions. The cingulate subregions were defined according to the Human Brainnetome Atlas based on connectional architecture and applying multimodal neuroimaging techniques (19). In each hemisphere, the CG was divided into the following subregions: CG-1 (dorsal area 23; A23d), CG-2 (rostroventral area 24; A24rv), CG-3 (pregenual area 32; A32p), CG-4 (ventral area 23; A23v), CG-5 (caudodorsal area 24; A24cd), CG-6 (caudal area 24; A23c) and CG-7 (subgenual area 32; A32sg). Thus, a total of 14 regions of interest (ROIs) were defined for the bilateral CG. The list of ROIs and the atlas details are displayed in Fig. 1 and Table I.

Functional connectivity analysis. The voxel-wise whole-brain FC maps for each of the cingulate subregions were investigated using the Dynamic BC software package (23). The average time series were extracted from each ROI. Subsequently, Pearson's correlation coefficients between the mean time courses of each ROI and the signal time series of each voxel in other parts of the brain were calculated. The results were subsequently converted to $\mathrm{z}$-values using Fisher's r-to-z transformation, to generate a Z-score FC map of improved normality (24). FC analysis was performed to identify aberrant FC characteristics for each ROI.

The ANT for executive control performance. Executive control performance was evaluated using the ANT. Stimuli in the ANT are visually displayed on a screen and participants are required to identify the direction in which a central target arrow points, as efficiently as possible. The reaction time (RT) of all participants was recorded. After all participants had completed a practice module of 24 trials, a formal test was performed, which included 3 modules comprising 96 trials each. A detailed description of the ANT has been published previously (25). The efficiency of the executive control network was determined as follows (26): Conflict effect $=\mathrm{RT}_{\text {incongruent }}-\mathrm{RT}_{\text {congruent }}$. A longer RT of conflict effect suggested inferior executive control performance.

Statistical analysis. Statistical analysis of the demographic and clinical data was performed using SPSS version 18.0 (SPSS, Inc.) and the Statistical Parametric Mapping software 


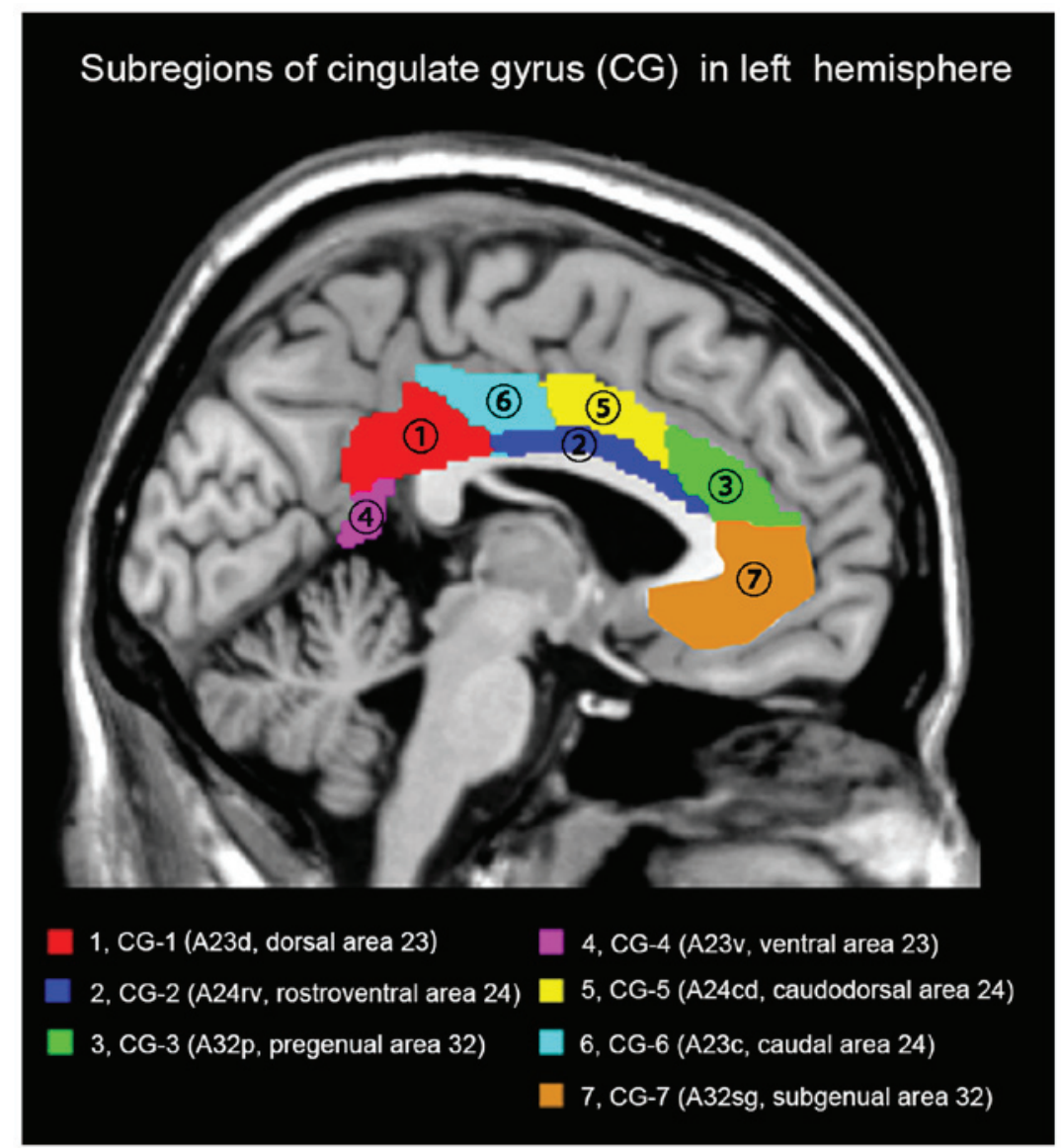

Figure 1. Regions of interest. Anatomical locations of the cingulate subregions used for functional connectivity analysis. CG, cingulate gyrus.

package (SPM12; http://www.fil.ion.ucl.ac.uk/spm) was used to analyze the rs-fMRI data. Differences in demographic data and clinical features between the two groups were analysed using an independent two-samples t-test and the sex distribution was analysed using the $\chi^{2}$ test. The differences in FC between the patient and control groups were compared using a two-samples t-tests in SPM12. Age, sex, education level and MMSE scores were used as covariates to be regressed in the two-sample t-tests (27). The results of statistical FC mapping were further corrected for multiple comparisons using Gaussian random fields (GRF) to a significant voxel level of $\mathrm{P}<0.001$, and a joint cluster level of $\mathrm{P}<0.01$, which was implemented using RESTplus software (http://resting-fmri.source forge.net).

In order to explore the association between the significant FC values and the clinical characteristics in the rTLE group, Pearson's correlation analysis was performed between the rsFC of the cingulate sub-regions and clinical variables of the patients (including executive control function and duration of disease). $\mathrm{P}<0.05$ was considered to indicate a statistically significant difference.

\section{Results}

Demographic and clinical data. The demographic and clinical data of all participants are summarized in Table II. No significant differences were present between the rTLE patients and HCs with regard to sex, age, education level or MMSE score.
Imaging data. The differences in the FC values between patients with rTLE and HCs were analysed for the 14 cingulate subregions (Table III). The FC maps of the left CG-1, bilateral CG-6, bilateral CG-7 and right CG-2 exhibited significant inter-group differences in connectivity [voxel-level threshold $\mathrm{P}<0.001$ and cluster-level threshold $\mathrm{P}<0.01$, GRF-corrected], as indicated in Fig. 2 (for regional details, see Table III). However, the FC values in the other 8 cingulate subregions were not significantly different between patients with rTLE and the HCs. Compared with that in the $\mathrm{HCs}$, one region in rTLE patients exhibited an enhanced FC, and five regions exhibited a decreased FC (primarily in the prefrontal cortex). Reduced FC values in patients with rTLE were detected in the following regions: i) Between the right CG-2 and the right superior frontal gyrus [SFG; extending to the right medial (m)SFG and the right medial frontal gyrus]; ii) between the left CG-6 and the right SFG (extending to the bilateral medial superior frontal gyrus); iii) between the right CG-6 and the right SFG [extending to the right middle frontal gyrus (MFG)]; iv) between the left CG-7 and the left SFG (extending to the left MFG, the right $\mathrm{mSFG}$ ); v) between the left CG-7 and the right SFG (extending to the right MFG and the bilateral supplementary motor area); and vi) between the right CG-7 and the left SFG (extending to the left MFG and the $\mathrm{mSFG}$ ). In addition, an increase in the FC value was observed between the left CG-1 and the left SFG (extending to the left MFG, the left supplementary motor area and the left MFG). 
Table II. Demographic information and clinical characteristics of patients with rTLE and healthy controls.

\begin{tabular}{|c|c|c|c|c|c|}
\hline Variable & $\begin{array}{c}\text { rTLE } \\
\text { group }(\mathrm{n}=27)\end{array}$ & $\begin{array}{c}\text { Control } \\
\text { group }(n=20)\end{array}$ & $\begin{array}{l}\text { Test statistic, } \\
\mathrm{t} / \chi^{2} \text {-value }\end{array}$ & df & P-value \\
\hline Sex & & & $\chi^{2}=0.648$ & 1 & 0.421 \\
\hline Male & $13(48)$ & $12(60)$ & & & \\
\hline Female & $14(52)$ & $8(49)$ & & & \\
\hline Right-handedness & $27(100)$ & $20(100)$ & N/A & N/A & N/A \\
\hline Age (years) & $29.89 \pm 11.41$ & $27.70 \pm 2.79$ & $\mathrm{t}=0.959$ & 30.11 & 0.345 \\
\hline Education (years) & $14.33 \pm 4.43$ & $16.25 \pm 3.47$ & $t=-1.605$ & 45.00 & 0.116 \\
\hline Duration of epilepsy (years) & $7.87 \pm 6.26$ & N/A & $\mathrm{N} / \mathrm{A}$ & N/A & N/A \\
\hline Mini-Mental-State Examination & $28.89 \pm 0.80$ & $28.80 \pm 0.77$ & $\mathrm{t}=0.383$ & 45.00 & 0.704 \\
\hline \multicolumn{6}{|l|}{$\mathrm{RT}(\mathrm{msec})$} \\
\hline Congruent & $622.72 \pm 109.82$ & $528.49 \pm 52.10$ & $t=3.905$ & 39.24 & $<0.001$ \\
\hline Incongruent & $745.48 \pm 120.82$ & $602.41 \pm 55.15$ & $\mathrm{t}=5.436$ & 38.51 & $<0.001$ \\
\hline Executive control (conflict effect) & $122.76 \pm 41.20$ & $73.92 \pm 20.59$ & $\mathrm{t}=5.327$ & 40.23 & $<0.001$ \\
\hline
\end{tabular}

Values are expressed as the mean \pm standard deviation or $\mathrm{n}(\%)$. RT, reaction time; rTLE, right-sided temporal lobe epilepsy; N/A, not applicable; df, degrees of freedom.

Table IV presents the statistical analysis of the FC z-scores using the cingulate subregions as ROIs. The results indicated that the mean z-scores of certain cingulate subregions in the rTLE group were lower than those for the HC group, whereas the mean $\mathrm{z}$-scores of other cingulate subregions were higher than those for the HCs. These were significantly different in cingulate subregions including the left CG-1, the left CG-3 and the left CG-7 (between patients with rTLE and HCs; P<0.05). These abnormal regions were more common in the left (three subregions) than in the right cingulate cortex.

Behavioral results during ANT performance. Compared with that in the $\mathrm{HC}$ group, patients with rTLE exhibited significantly poorer accuracy when assessing congruent and incongruent conditions $(\mathrm{P}<0.001$; Fig. 3 and Table II). Furthermore, the executive control efficiency (conflict effect) RT of the patients was significantly longer than that from the $\mathrm{HC}$ group $(\mathrm{P}<0.05$; Fig. 3 and Table II).

Correlation between abnormal FC and clinical characteristics. A correlation analysis was performed between the altered cingulate subregions with significantly different FC values and clinical characteristics (including executive control performance) in patients with rTLE. Pearson's correlation analysis revealed that the $\mathrm{zFC}$ values of the left CG-1 and left SFG were negatively correlated with the conflict effect ( $\mathrm{r}=-0.45$; $\mathrm{P}=0.019$, uncorrected) (Fig. 4). The other cingulate subregions with abnormal FC values were not significantly correlated with executive control performance or any other clinical variables. Furthermore, no significant correlation was obtained within the HC group.

\section{Discussion}

To the best of our knowledge, the present study was the first to use rs-fMRI to systematically investigate alterations in the rsFC of cingulate subregions in rTLE. It was revealed that patients with rTLE had altered patterns of connectivity between different cingulate subregions and cortical brain regions (primarily in the prefrontal lobe). The patients also exhibited executive control impairment. The number of cingulate subregions with statistically significant FC changes was slightly higher in the left than in the right cingulate cortex, and the FC variations of the cingulate subregions were determined to be specific to rTLE. It was also indicated that increased connectivity in the left CG-1 and left SFG were negatively correlated with executive control performance in rTLE.

Due to its cytoarchitectural and functional framework, the cingulate cortex is considered to be a complex brain structure $(10,19)$, and is divided into four subregions based on function and anatomy (a popular means of classification) (17). Numerous studies have identified that the ACC has a predominant role in control, monitoring, executive control of attention (28) and affective functions (29); the ACC is associated with the frontal and limbic regions of the brain, which are associated with these functions, particularly in the prefrontal cortex (30). The MCC is engaged in decision-making and multisensory orientation, as well as conflict monitoring. The PCC is an important interactive core between the DMN and the sensorimotor network (31), which is also associated with the precuneus, anterior and medial regions of the SFG $(32,33)$. The RSCC is located at the interface of the ACC, MCC and PCC, and is associated with the parahippocampal memory system. According to the Human Brainnetome Atlas (19), which was utilized in the present study, the CG-7 and the CG-3 correspond to the ACC. In addition, the locations of the CG-6, the CG-5 and the CG-2 in the present study are akin to that of the MCC in previous studies $(34,35)$. Furthermore, the CG-1 in the present study belongs to the PCC and the CG-4 is located deep within the CG and corresponds to the RSCC.

As the anterior part of the cingulate cortex, the CG-7 is one of the key regions of the dorsal attention network, which is active during cognitive tasks (36). The ACC, which is 


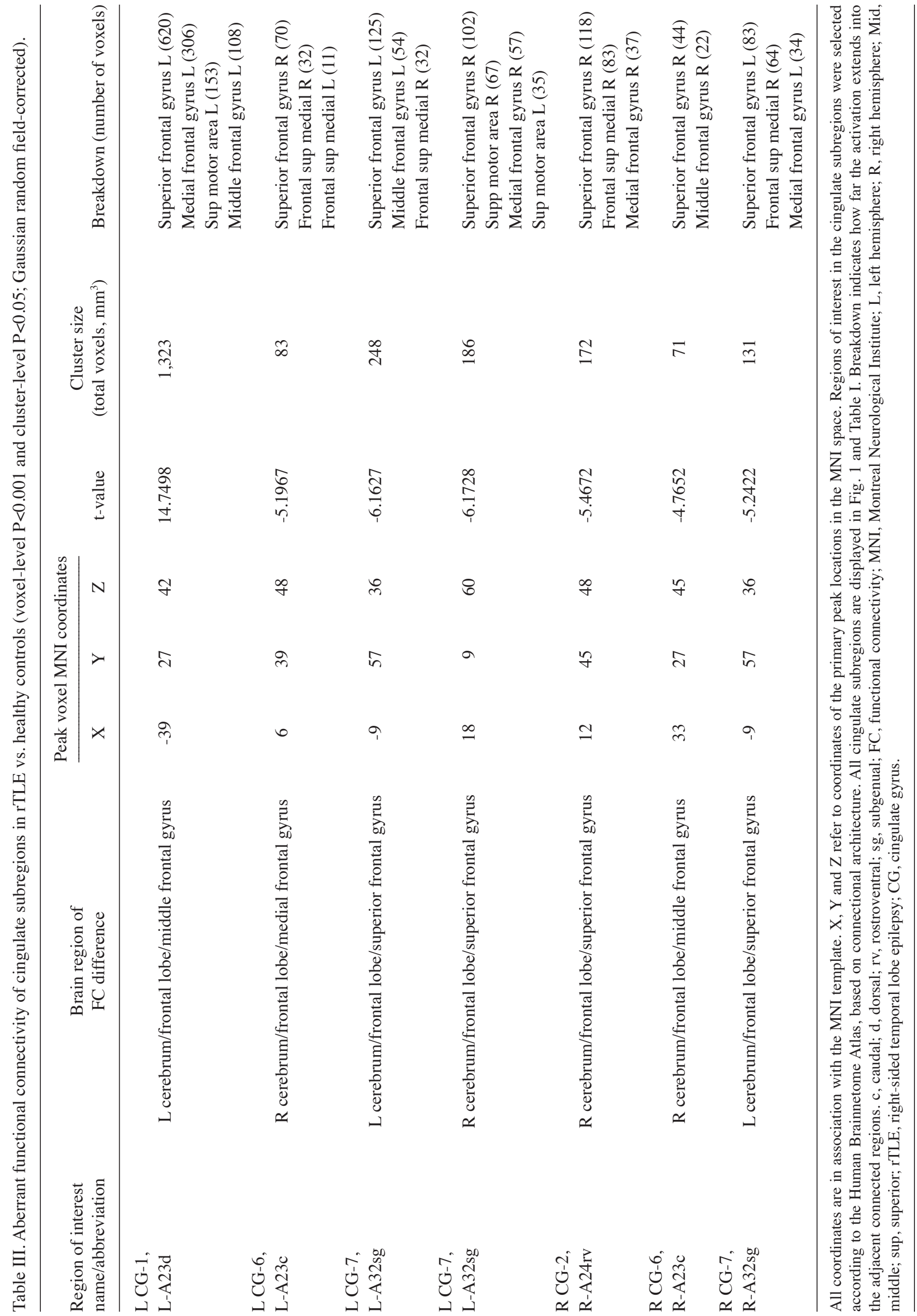




\section{Left_ROls}

CG-1 (A23d, dorsal area 23) $R$
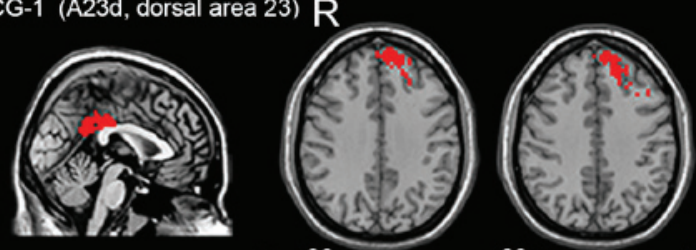

$+39 \mathrm{~mm}$

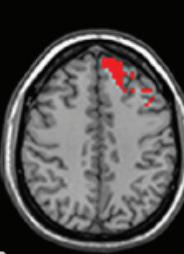

$+42 \mathrm{~mm}$

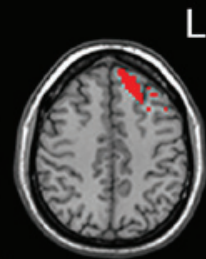

$+45 \mathrm{~mm}$

CG-6 (A23c, caudal area 24)
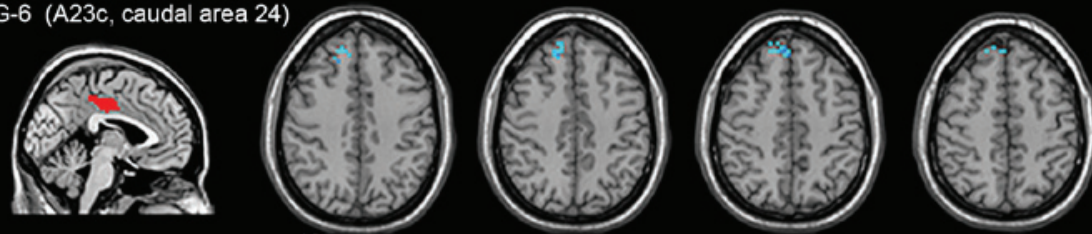

$\mathrm{z}=+42 \mathrm{~mm}$

$+45 \mathrm{~mm}$

$+48 \mathrm{~mm}$

$+51 \mathrm{~mm}$

CG-7 (A32sg, subgenual area 32)
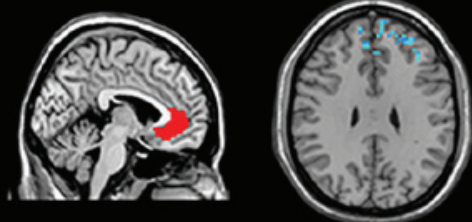

$\mathrm{z}=-43 \mathrm{~mm}$
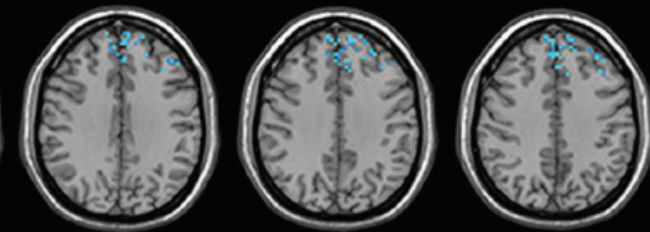

$-27 \mathrm{~mm}$

$-19 \mathrm{~mm}$

CG-7 (A32sg, subgenual area 32)
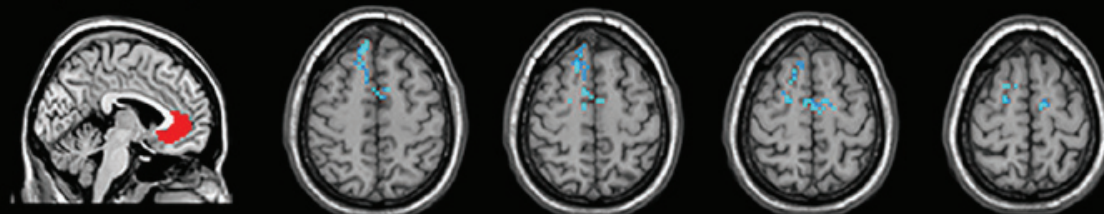

\section{Right_ROls}

CG-2 (A24rv, rostroventral area 24)
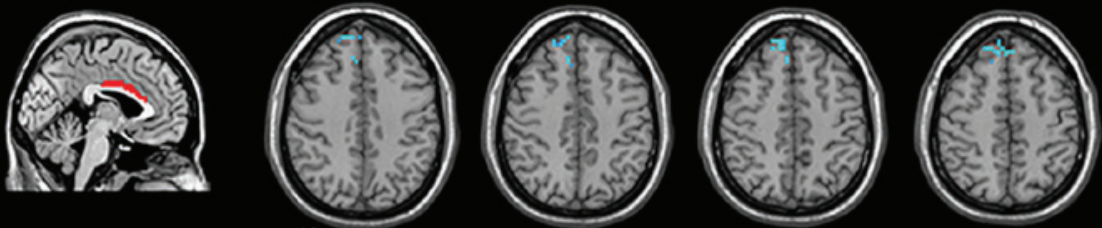

$\mathrm{z}=+42 \mathrm{~mm}$

$+45 \mathrm{~mm}$

$+48 \mathrm{~mm}$

$+51 \mathrm{~mm}$

CG-6 (A23C, caudal area 24)
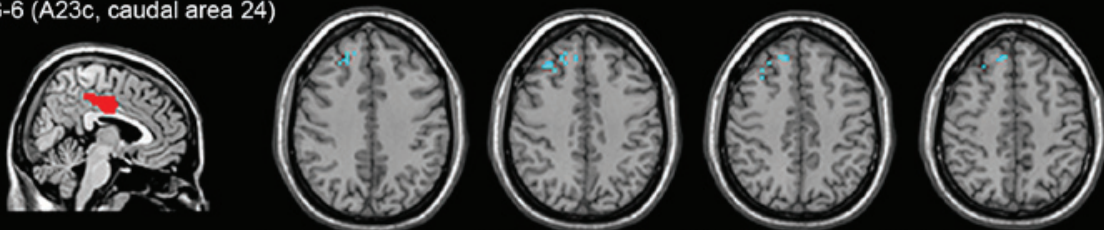

+42 mm

$+45 \mathrm{~mm}$

$+48 \mathrm{~mm}$

CG-7 (A32sg, Subgenual area 32)
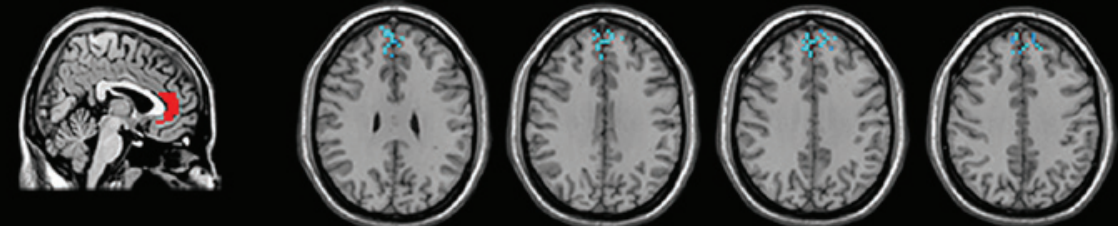

$\mathrm{z}=+30 \mathrm{~mm}$

$+33 \mathrm{~mm}$

$+36 \mathrm{~mm}$

+39 mm

$-2$

30

$-6$

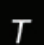

Figure 2. Statistical maps indicating the significant differences in FC of the cingulate subregions in patients with rTLE, compared with healthy controls. Warm colors indicate increased FC values and cool colors denote decreased FC values in patients with rTLE; the color bar represents the t-values from two-samples t-tests; $\mathrm{z}$ (with an incremental interval of 3 or 8 ) represents the $\mathrm{z}$-axis for the Montreal Neurological Institute coordinates. Voxel-level threshold $\mathrm{P}<0.001$ and cluster-level threshold $\mathrm{P}<0.01$; Gaussian random field-corrected. rTLE, right-sided temporal lobe epilepsy; FC, functional connectivity; ROI, region of interest; GC, cingulate gyrus. 
Table IV. Results of FC z-scores extracted using cingulate subregions as regions of interest.

\begin{tabular}{|c|c|c|c|c|c|c|}
\hline \multirow[b]{2}{*}{ Label ID } & \multirow[b]{2}{*}{ Subregion abbreviation } & \multicolumn{2}{|c|}{ z-score ${ }^{a}$} & \multicolumn{3}{|c|}{ Statistical parameters ${ }^{b}$} \\
\hline & & Subjects $(n=27)$ & Controls $(n=20)$ & P-value ${ }^{c}$ & F value & P-value ${ }^{d}$ \\
\hline CG-1 & L-A23d & $1.148 \pm 0.199$ & $0.357 \pm 0.167$ & 0.780 & 0.079 & $0.000^{\mathrm{e}}$ \\
\hline CG-2 & L-A24rv & $0.902 \pm 0.282$ & $0.997 \pm 0.248$ & 0.686 & 0.165 & 0.238 \\
\hline CG-3 & L-A32p & $1.003 \pm 0.168$ & $1.112 \pm 0.181$ & 0.870 & 0.027 & $0.038^{\mathrm{e}}$ \\
\hline CG-4 & L-A23v & $1.069 \pm 0.203$ & $1.090 \pm 0.138$ & 0.035 & 4.701 & 0.675 \\
\hline CG-5 & L-A24cd & $1.080 \pm 0.216$ & $1.127 \pm 0.210$ & 0.878 & 0.024 & 0.455 \\
\hline CG-6 & L-A23c & $0.985 \pm 0.166$ & $1.093 \pm 0.214$ & 0.264 & 1.280 & 0.055 \\
\hline $\mathrm{CG}-7$ & L-A32sg & $0.999 \pm 0.196$ & $1.113 \pm 0.161$ & 0.199 & 1.698 & $0.039^{\mathrm{e}}$ \\
\hline CG-1 & R-A23d & $1.121 \pm 0.183$ & $1.182 \pm 0.215$ & 0.457 & 0.562 & 0.300 \\
\hline CG-2 & R-A24rv & $0.782 \pm 0.168$ & $0.884 \pm 0.182$ & 0.405 & 0.706 & 0.054 \\
\hline CG-3 & R-A32p & $1.187 \pm 0.149$ & $1.252 \pm 0.202$ & 0.115 & 2.583 & 0.211 \\
\hline CG-4 & $\mathrm{R}-\mathrm{A} 23 \mathrm{v}$ & $1.009 \pm 0.120$ & $1.028 \pm 0.140$ & 0.195 & 1.732 & 0.723 \\
\hline CG-5 & R-A24cd & $1.235 \pm 0.204$ & $1.248 \pm 0.185$ & 0.598 & 0.282 & 0.829 \\
\hline CG-6 & $\mathrm{R}-\mathrm{A} 23 \mathrm{c}$ & $0.931 \pm 0.173$ & $0.951 \pm 0.145$ & 0.309 & 1.059 & 0.683 \\
\hline CG-7 & R-A32sg & $1.136 \pm 0.204$ & $1.238 \pm 0.185$ & 0.736 & 0.115 & 0.083 \\
\hline
\end{tabular}

${ }^{\mathrm{a}} \mathrm{Z}$-score data presented as the mean \pm standard deviation; ${ }^{\mathrm{b}}$ Analysis of covariance adjusted for age, sex and years of education; ${ }^{\mathrm{c}} \mathrm{P}$-values representing homogeneity of variance; ${ }^{\mathrm{d}} \mathrm{P}$-values for analysis of covariance; ${ }^{\mathrm{e}}$ significant $\mathrm{P}$-values following analysis of covariance $(\mathrm{P}<0.05)$ and assessment of homogeneity of variance $(\mathrm{P}>0.05)$. L, left hemisphere; R, right hemisphere; FC, functional connectivity; CG, cingulate gyrus .

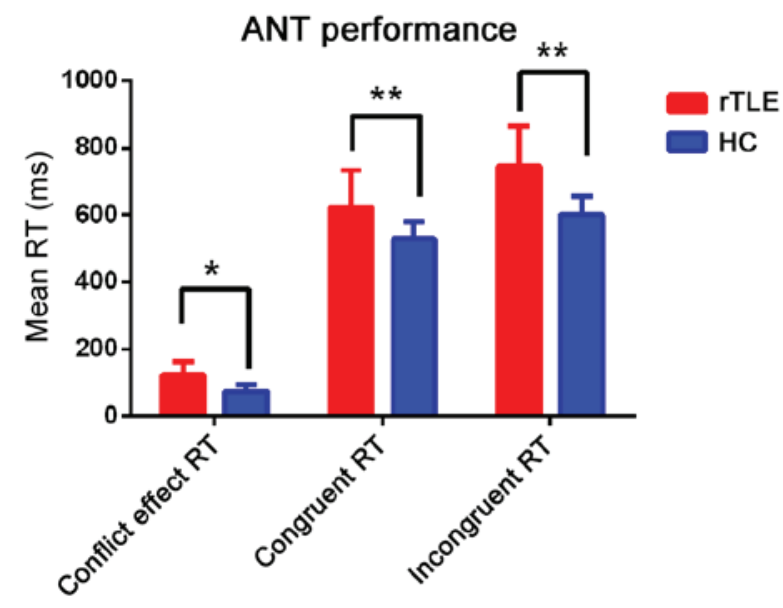

Figure 3. ANT results for patients with rTLE and HCs. Values are expressed as the mean \pm standard deviation. ${ }^{*} \mathrm{P}<0.05$ and ${ }^{* *} \mathrm{P}<0.01$. ANT, attention network test; RT, reaction time; rTLE, right-sided temporal lobe epilepsy; $\mathrm{HC}$, healthy control; ms, millisecond.

associated with high-level cognitive and emotional processing, has distinct connections with various cortical areas, including the prefrontal cortex (37). The anterior portion of the ACC has also been viewed as 'executive' in function (38), and in line with previous studies, the CG-7 may be involved in monitoring the response to conflict (39). In the present study, patients with rTLE showed reduced CG-7-SFG connectivity compared with HCs. The SFG has been reported to be associated with cognitive and motor control processes, execution and attention (40). Another study suggested that TLE with executive control impairment resulted in hypoperfusion in

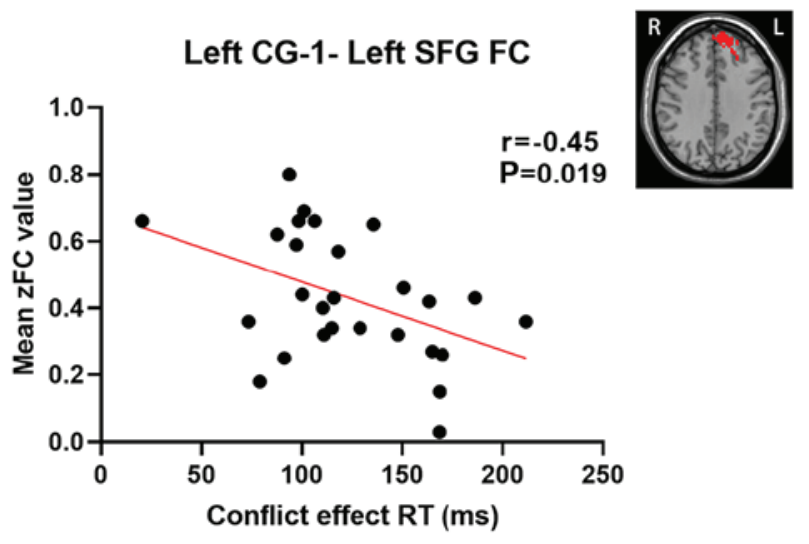

Figure 4. Significant negative correlation between increased left CG-1-left SFG FC and executive control performance in patients with rTLE. The conflict effect represents executive control efficiency. Red indicates increased functional connectivity in the left CG-1 and left SFG in patients with rTLE compared with healthy controls. $\mathrm{P}<0.05$, uncorrected. CG-1, cingulate gyrus-1 (dorsal area 23; A23d); SFG, superior frontal gyrus; FC, functional connectivity; ms, millisecond; RT, reaction time; L, left hemisphere; R, right hemisphere.

the extratemporal areas (including the SFG) in patients with TLE (41). Another fMRI study revealed decreased FC of the affected hippocampus and SFG in patients with TLE (42). A reduction in $\mathrm{FC}$ was also reported within the right superior frontal lobe (BA 8), though FC enhancement was observed within the right superior frontal lobe (BA 11) in patients with rTLE when performing the Visuospatial Working Memory N-back task (43). In addition, decreased FC between the ACC and the frontal lobe has been reported in patients with generalized tonic-clonic seizure epilepsy, and is associated with 
cognition impairment (44). Execution control activities are frequently associated with the frontal cortex, particularly in the ACC and prefrontal cortex (45). The results of the present study are consistent with those of previous studies, suggesting that decreased FC of the bilateral CG-7 and the bilateral SFG may be one of the underlying mechanisms of executive control impairment.

The CG-2 and the CG- 6 belong to the middle region of the cingulate cortex, and are involved in feedback-mediated decision making and attention monitoring (46). According to the theory of Vogt, these regions are associated with modulating cognitive motor responses and integrating various sources from cognitive and emotional networks, which improves decision-making efficiency and execution (34). The present study revealed that the rTLE group exhibited reduced FC in the right CG-2 and the right SFG, as well as decreased FC between the bilateral CG-6 and the right SFG, with stronger connections to the prefrontal lobe.

The SFG is considered to be part of the DMN and evidence indicates that they may have a crucial role in goal-directed behavior by regulating the connection between the DMN and the fronto-parietal control network (47). The SFG has been reported to be strongly associated with cognitive control functions, including executive control (40). A previous study suggested that significant cortical thinning was present in the bilateral sensorimotor cortex, including in the bilateral SFG in patients with TLE (48). Afzali et al (49), performed a diffusion tensor imaging study and revealed that the SFG and MFG are regions with reduced fractional anisotropy in patients with TLE, suggesting that the microstructure of white matter was impaired. In patients with TLE, abnormalities of dynamic functional network interactions were identified between the left superior medial frontal cortex and the left middle frontal gyrus, which indicated that network modulation failure may be associated with cognitive impairment in epilepsy (50). The results of the present study are consistent with those of previous studies, indicating that this disruption in connectivity may be linked to executive control impairment in rTLE.

The CG-1 belongs to the posterior region of the cingulate cortex and is considered to be a hub region within the DMN (32). The DMN is characterized by its maintenance of brain activity in the resting state (when the individual is not focused) and exhibits decreased activity during attention-demanding tasks (51). Disruptions of the functional and structural connectivity between the DMN have been reported in TLE (52), which may promote the cognitive impairments associated with TLE (53-55). Using probabilistic fiber tracking, Khalsa et al (56) investigated the underlying structural connectivity within the DMN and demonstrated stronger structural connections between the PCC and the mesial prefrontal cortex in normal subjects. Evidence suggests that the SFG, the superior region of the prefrontal cortex, is composed of several different subregions, which account for its diverse connection patterns and specific function (40). Koshino et al (57) observed increased activation of the medial prefrontal cortex and the posterior cingulate cortex during task preparation, suggesting that according to the task requirements, the allocation of attentional resources to the task-associated areas affected the activation of these network sites. Another study indicated that increased connectivity between the left superior frontal gyrus and the posterior cingulate cortex predicted faster RT during task performance. In addition, the interaction and dynamic reconfiguration in functional connections of the DMN indicates that they are involved in the processing of current tasks at a higher global level (33). The present study indicated increased connectivity between the left CG-1 and the left SFG in rTLE, compared with that in the $\mathrm{HC}$ group, which is in keeping with the results of previous studies (58). The increased FC in these regions may be interpreted as a compensation mechanism for executive control deficits (59).

Attention deficit is one of the most common symptoms of TLE (60). Executive control is an important component of attention but is frequently neglected and is rarely reported in TLE. The executive control network governs the capacity to achieve intended goals and responses to resolve conflict. In the present study, executive control was evaluated by a flanker trial where participants were asked to identify the direction of a central arrow, which was 'flanked' by congruent or incongruent stimuli (arrows that are the same or opposed to the target direction, respectively). When the target arrow and the flanking arrows are congruent, participants typically respond faster than when the arrows are incongruent. The results suggested that patients with TTLE and HCs responded more rapidly during congruent than during incongruent flanker conditions, suggesting a typical flanker congruent efficiency. Of note, patients with rTLE exhibited longer RTs for task-irrelevant stimuli than the HCs, which indicates that patients with rTLE have difficulties in resolving the conflict between task-relevant and task-irrelevant stimuli. The RT of the executive control network in patients with rTLE was significantly longer than that of the HCs. This indicates that patients with rTLE took longer to resolve conflict than the HCs, suggesting that the executive control network efficiency of patients with rTLE is lower. The results of the present study revealed poorer executive control performance in patients with rTLE, which is in line with a previous study (61). The results also demonstrated a moderate but significant negative correlation between the executive control performance and the abnormal zFC between the left CG-1 and left SFG in patients with rTLE. The results of a previous functional neuroimaging study suggested that prefrontal areas, including the SFG, are involved in response conflicts and response errors. A previous study supported that the SFG is part of the DMN and may mediate the connection between the default network and the fronto-parietal control network to facilitate goal-directed executive processes (47).

The results of the present study are consistent with those of a previous study in which increased connectivity between the PCC and medial prefrontal cortex was associated with improved working memory performance (62). The present study suggested that the left SFG may serve a potential compensatory role in executive processing in rTLE, which is in line with the previous studies $(60,63)$. However, due to the small sample size, the results of the correlation analysis were not corrected for multiple comparisons, and hence, the results should be considered preliminary. In addition, the brain regions exhibiting FC variations (using the left CG-1, CG-3 and CG-7 as the seed point) were located in the left hemisphere. There appears to be no reasonable interpretation for these results and this observation may be attributed to the altered structure of the CG; additional structural research is required to confirm this hypothesis. The total number of cingulate subregions with 
significant FC alterations was also higher in the left than in the right $\mathrm{CG}$, indicating potential left $\mathrm{CG}$ dominance. A previous study used the ANT to investigate hemisphere dominance for the executive control network and indicated greater executive control efficiency in the right hemisphere relative to the left, regarding the reaction time (64). Another rs-fMRI study based on graph theory examined the differences in limbic network connectivity between left-sided TLE (ITLE) and rTLE, revealing a more significant reduction in limbic network connectivity in rTLE than in ITLE (65). The results of the aforementioned studies are inconsistent with those of the present study, which may be due to the heterogeneity of the CG, as well as differences in sample size and analysis methods. However, further studies are required to confirm this speculation.

The present study had several limitations. First, the patients were treated with different anti-epileptic drugs at the time of recruitment, which may have exerted different effects on the brain. The possible effects of anti-epileptic therapy on FC analysis cannot be excluded. Furthermore, the small sample size may have resulted in reduced statistical power; in addition, only rTLE was investigated and the absence of a ITLE group may have limited the results of the study. Furthermore, the small sample size prevented any subgroup analyses and studies with larger samples are required to validate the present results. Although various results were consistent with the Brainnetome Atlas, it should be noted that the underlying inter-individual variability of the cingulate subregions may have influenced the results of the present study (19). Finally, the present study only used rs-FC to investigate alterations to the cingulate subregions in rTLE; in future studies, multimodal data fusion analysis will be performed to explore abnormalities in cingulate subregion connectivity in TLE, and as such, the present results should be considered as preliminary data and treated with caution.

To the best of our knowledge, the present study was the first to systematically investigate the rsFC patterns of the cingulate subregions in patients with rTLE. Executive control dysfunction was observed in patients with rTLE, compared with the HCs. Significant alterations in $\mathrm{rsFC}$ between the cingulate subregion and the brain regions were mostly decreased (though some were slightly increased), suggesting that FC may have a left-side advantage in patients with rTLE. The FC variations of the cingulate subregions were reported to be specific to rTLE. Furthermore, the correlation between the altered FC values and executive control performance suggests a compensatory mechanism on executive control deficits in pathological conditions. The information on the differentially altered functional connectivity patterns of cingulate subregions revealed in the present study may provide a deeper understanding of the complex neurological mechanisms and executive control dysfunctions underlying rTLE.

\section{Acknowledgements}

Not applicable.

\section{Funding}

The present study was supported by The National Natural Science Foundation of China (grant no. 81560223) and Guangxi Natural Science Foundation (grant no.2018GXNSFAA050149).

\section{Availability of data and materials}

The datasets used and/or analysed during the current study are available from the corresponding author upon reasonable request.

\section{Authors' contributions}

$\mathrm{ZZ}$ and $\mathrm{JZ}$ conceived and designed the research study. XZ, JL, LQ and WY performed the research. ZZ, XZ and LQ analysed the data. XZ, JL and WY supervised the data analysis. ZZ wrote the original draft. JZ, XZ, JL, WY and LQ reviewed and edited the manuscript. All authors read and approved the manuscript.

\section{Ethics approval and informed consent to participate}

All subjects were informed in detail about the research and they provided written informed consent. The study was approved by The Ethics Committee of the First Affiliated Hospital of Guangxi Medical University (Nanning, China).

\section{Patient consent for publication}

Not applicable.

\section{Competing interests}

The authors declare that they have no competing interests.

\section{References}

1. Behr C, Goltzene M, Kosmalski G, Hirsch E and Ryvlin P: Epidemiology of epilepsy. Rev Neurol (Paris) 172: 27-36, 2016.

2. Téllez-Zenteno JF and Hernández-Ronquillo L: A review of the epidemiology of temporal lobe epilepsy. Epilepsy Res Treat 2012: 630853, 2012.

3. Jo HJ, Kenney-Jung DL, Balzekas I, Welker KM, Jones DT, Croarkin PE, Benarroch EE and Worrell GA: Relationship between seizure frequency and functional abnormalities in limbic network of medial temporal lobe epilepsy. Front Neurol 10: 488, 2019.

4. Urbach H, Egger K, Rutkowski K, Nakagawa JM, Schmeiser B, Reisert M, Brandt A, Steinhoff BJ, Schulze-Bonhage A and Hammen T: Bilateral cingulum fiber reductions in temporal lobe epilepsy with unilateral hippocampal sclerosis. Eur J Radiol 94: 53-57, 2017

5. Guo L, Bai G, Zhang H, Lu D, Zheng J and Xu G: Cognitive functioning in temporal lobe epilepsy: A BOLD-fMRI study. Mol Neurobiol 54: 8361-8369, 2017.

6. Coan AC, Appenzeller S, Bonilha L, Li LM and Cendes F: Seizure frequency and lateralization affect progression of atrophy in temporal lobe epilepsy. Neurology 73: 834-842, 2009.

7. Fox MD, Snyder AZ, Vincent JL, Corbetta M, Van Essen DC and Raichle ME: The human brain is intrinsically organized into dynamic, anticorrelated functional networks. Proc Natl Acad Sci USA 102: 9673-9678, 2005.

8. Yu C, Zhou Y, Liu Y, Jiang T, Dong H, Zhang Y and Walter M: Functional segregation of the human cingulate cortex is confirmed by functional connectivity based neuroanatomical parcellation. Neuroimage 54: 2571-2581, 2011.

9. Peterson BS, Skudlarski P, Gatenby JC, Zhang H, Anderson AW and Gore JC: An fMRI study of Stroop word-color interference: Evidence for cingulate subregions subserving multiple distributed attentional systems. Biol Psychiatry 45: 1237-1258, 1999.

10. Shackman AJ, Salomons TV, Slagter HA, Fox AS, Winter JJ and Davidson RJ: The integration of negative affect, pain and cognitive control in the cingulate cortex. Nat Rev Neurosci 12: 154-167, 2011. 
11. Torta DM and Cauda F: Different functions in the cingulate cortex, a meta-analytic connectivity modeling study. Neuroimage 56 $2157-2172,2011$

12. Narenmandula B, Zhou X, Li Y, Tu D, Bao Y, Zheng R and $\mathrm{Xu} \mathrm{H}$ : Effects of white matter microstructure lesions on language and memory function in magnetic resonance imaging-negative temporal lobe epilepsy determined by diffusion tensor imaging. Neurol India 64: 1233-1242, 2016.

13. Kojan M, Doležalová I, Korit’áková E, Mareček R, Řehák Z, Hermanová M, Brázdil $M$ and Rektor I: Predictive value of preoperative statistical parametric mapping of regional glucose metabolism in mesial temporal lobe epilepsy with hippocampal sclerosis. Epilepsy Behav 79: 46-52, 2018.

14. Zeng H, Pizarro R, Nair VA, La C and Prabhakaran V: Alterations in regional homogeneity of resting-state brain activity in mesial temporal lobe epilepsy. Epilepsia 54: 658-666, 2013.

15. Chiang S, Stern JM,Engel J Jr and Haneef Z: Structural-functional coupling changes in temporal lobe epilepsy. Brain Res 1616 : 45-57, 2015.

16. Vogt BA, Nimchinsky EA, Vogt LJ and Hof PR: Human cingulate cortex: Surface features, flat maps, and cytoarchitecture. J Comp Neurol 359: 490-506, 1995.

17. Vogt BA: Pain and emotion interactions in subregions of the cingulate gyrus. Nat Rev Neurosci 6: 533-544, 2005

18. Beckmann M, Johansen-Berg $\mathrm{H}$ and Rushworth MF: Connectivity-based parcellation of human cingulate cortex and its relation to functional specialization. J Neurosci 29: 1175-1190, 2009.

19. Fan L, Li H, Zhuo J, Zhang Y, Wang J, Chen L, Yang Z, Chu C, Xie S, Laird AR, et al: The human brainnetome atlas: A new brain atlas based on connectional architecture. Cereb Cortex 26 3508-3526, 2016

20. Fan J, McCandliss BD, Sommer T, Raz A and Posner MI: Testing the efficiency and independence of attentional networks. J Cogn Neurosci 14: 340-347, 2002.

21. Manford M, Fish DR and Shorvon SD: An analysis of clinical seizure patterns and their localizing value in frontal and temporal lobe epilepsies. Brain 119: 17-40, 1996.

22. Yan CG, Wang XD, Zuo XN and Zang YF: DPABI: Data processing and analysis for (Resting-State) brain imaging. Neuroinformatics 14: 339-351, 2016.

23. Liao W, Wu GR, Xu Q, Ji GJ, Zhang Z, Zang YF and Lu G: DynamicBC: A MATLAB toolbox for dynamic brain connectome analysis. Brain Connect 4: 780-790, 2014

24. Lowe MJ, Mock BJ and Sorenson JA: Functional connectivity in single and multislice echoplanar imaging using resting-state fluctuations. Neuroimage 7: 119-132, 1998.

25. Li J, Chen X, Ye W, Jiang W, Liu H and Zheng J: Alteration of the alertness-related network in patients with right temporal lobe epilepsy: A resting state fMRI study. Epilepsy Res 127: 252-259, 2016.

26. Fan J, McCandliss BD, Fossella J, Flombaum JI and Posner MI: The activation of attentional networks. Neuroimage 26: 471-479, 2005.

27. Li R, Liu K, Ma X, Li Z, Duan X, An D, Gong Q, Zhou D and Chen H: Altered functional connectivity patterns of the insular subregions in psychogenic nonepileptic seizures. Brain Topogr 28: 636-645, 2015 .

28. Heilbronner SR and Hayden BY: Dorsal anterior cingulate cortex: A bottom-up view. Annu Rev Neurosci 39: 149-170, 2016

29. Braem S, King JA, Korb FM, Krebs RM, Notebaert W and Egner T: The role of anterior cingulate cortex in the affective evaluation of conflict. J Cogn Neurosci 29: 137-149, 2017.

30. Boschin EA, Brkic MM, Simons JS and Buckley MJ: Distinct roles for the anterior cingulate and dorsolateral prefronta cortices during conflict between abstract rules. Cereb Cortex 27 34-45, 2017.

31. Cui F, Zhou L, Wang Z, Lang C, Park J, Tan Z, Yu Y, Sun C, Gao Y and Kong J: Altered functional connectivity of striata subregions in patients with multiple sclerosis. Front Neurol 8 129, 2017.

32. Yang R, Gao C, Wu X, Yang J, Li S and Cheng H: Decreased functional connectivity to posterior cingulate cortex in major depressive disorder. Psychiatry Res Neuroimaging 255: 15-23, 2016.

33. Vatansever D, Menon DK, Manktelow AE, Sahakian BJ and Stamatakis EA: Default mode network connectivity during task execution. Neuroimage 122: 96-104, 2015.

34. Vogt BA: Midcingulate cortex: Structure, connections, homologies, functions and diseases. J Chem Neuroanat 74: 28-46, 2016
35. Caruana F, Gerbella M, Avanzini P, Gozzo F, Pelliccia V, Mai R, Abdollahi RO, Cardinale F, Sartori I, Lo Russo G and Rizzolatti G: Motor and emotional behaviours elicited by electrical stimulation of the human cingulate cortex. Brain 141: 3035-3051, 2018

36. Fox MD, Corbetta M, Snyder AZ, Vincent JL and Raichle ME Spontaneous neuronal activity distinguishes human dorsal and ventral attention systems. Proc Natl Acad Sci USA 103: 10046-10051, 2006.

37. Bush G, Luu P and Posner MI: Cognitive and emotional influences in anterior cingulate cortex. Trends Cogn Sci 4: 215-222, 2000.

38. Vogt BA, Finch DM and Olson CR: Functional heterogeneity in cingulate cortex: The anterior executive and posterior evaluative regions. Cereb Cortex 2: 435-443, 1992.

39. Breton F, Planté A, Legauffre C, Morel N, Ades J, Gorwood P, Ramoz $\mathrm{N}$ and Dubertret C: The executive control of attention differentiates patients with schizophrenia, their first-degree relatives and healthy controls. Neuropsychologia 49: 203-208, 2011.

40. Li W, Qin W, Liu H, Fan L, Wang J, Jiang T and Yu C: Subregions of the human superior frontal gyrus and their connections. Neuroimage 78: 46-58, 2013.

41. Dupont P, Zaknun JJ, Maes A, Tepmongkol S, Vasquez S, Bal CS, Van Paesschen W, Carpintiero S, Locharernkul C and Dondi M: Dynamic perfusion patterns in temporal lobe epilepsy. Eur J Nucl Med Mol Imaging 36: 823-830, 2009.

42. Pittau F, Grova C, Moeller F, Dubeau F and Gotman J: Patterns of altered functional connectivity in mesial temporal lobe epilepsy. Epilepsia 53: 1013-1023, 2012.

43. Lv ZX, Huang DH, Ye W, Chen ZR, Huang WL and Zheng JO: Alteration of functional connectivity within visuospatial working memory-related brain network in patients with right temporal lobe epilepsy: A resting-state fMRI study. Epilepsy Behav 35 64-71, 2014

44. Ke M, Jin B, Liu G and Yang X: Impairments of cingulated cortex in the generalized tonic-clonic seizure epilepsy by combining morphological and functional connectivity magnetic resonance imaging. J Integr Neurosci 16: 429-439, 2017.

45. Casey BJ, Thomas KM, Welsh TF, Badgaiyan RD, Eccard CH, Jennings JR and Crone EA: Dissociation of response conflict, attentional selection, and expectancy with functional magnetic resonance imaging. Proc Natl Acad Sci USA 97: 8728-8733, 2000.

46. Pardo JV, Fox PT and Raichle ME: Localization of a human system for sustained attention by positron emission tomography. Nature 349: 61-64, 1991

47. Spreng RN, Stevens WD, Chamberlain JP, Gilmore AW and Schacter DL: Default network activity, coupled with the frontoparietal control network, supports goal-directed cognition. Neuroimage 53: 303-317, 2010.

48. Labate A, Cerasa A, Aguglia U, Mumoli L, Quattrone A and Gambardella A: Neocortical thinning in 'benign' mesial temporal lobe epilepsy. Epilepsia 52: 712-717, 2011.

49. Afzali M, Soltanian-Zadeh $\mathrm{H}$ and Elisevich KV: Tract based spatial statistical analysis and voxel based morphometry of diffusion indices in temporal lobe epilepsy. Comput Biol Med 41: 1082-1091, 2011.

50. Tailby C, Kowalczyk MA and Jackson GD: Cognitive impairment in epilepsy: The role of reduced network flexibility. Ann Clin Transl Neurol 5: 29-40, 2017.

51. Raichle ME: The brain's default mode network. Annu Rev Neurosci 38: 433-447, 2015.

52. Haneef Z, Lenartowicz A, Yeh HJ, Engel J Jr and Stern JM: Effect of lateralized temporal lobe epilepsy on the default mode network. Epilepsy Behav 25: 350-357, 2012.

53. Hsiao FJ, Yu HY, Chen WT, Kwan SY, Chen C, Yen DJ, Yiu CH, Shih YH and Lin YY: Increased intrinsic connectivity of the default mode network in temporal lobe epilepsy: Evidence from resting-state MEG recordings. PLoS One 10: e0128787, 2015.

54. Yang H, Zhang C, Liu C, Yu T, Zhang G, Chen N and Li K: Brain network alteration in patients with temporal lobe epilepsy with cognitive impairment. Epilepsy Behav 81: 41-48, 2018.

55. Liu M, Bernhardt BC, Hong SJ, Caldairou B, Bernasconi A and Bernasconi N: The superficial white matter in temporal lobe epilepsy: A key link between structural and functional network disruptions. Brain 139: 2431-2440, 2016.

56. Khalsa S, Mayhew SD, Chechlacz M, Bagary M and Bagshaw AP: The structural and functional connectivity of the posterior cingulate cortex: Comparison between deterministic and probabilistic tractography for the investigation of structure-function relationships. Neuroimage 102: 118-127, 2014. 
57. Koshino H, Minamoto T, Yaoi K, Osaka M and Osaka N: Coactivation of the default mode Network regions and Working Memory Network regions during task preparation. Sci Rep 4: 5954,2014

58. Zhou X, Zhang Z, Liu J, Qin L and Zheng J: Aberrant topological organization of the default mode network in temporal lobe epilepsy revealed by graph-theoretical analysis. Neurosci Lett 708: 134351, 2019.

59. Haneef Z, Lenartowicz A, Yeh HJ, Engel J Jr and Stern JM Network analysis of the default mode network using functional connectivity MRI in temporal lobe epilepsy. J Vis Exp 90: e51442, 2014.

60. Zhang Z, Lu G, Zhong Y, Tan Q, Yang Z, Liao W, Chen Z, Shi J and Liu Y: Impaired attention network in temporal lobe epilepsy: A resting FMRI study. Neurosci Lett 458: 97-101, 2009.

61. Li X, Hou Y, Ren Y, Tian X and Song Y: Alterations of theta oscillation in executive control in temporal lobe epilepsy patients. Epilepsy Res 140: 148-154, 2018.
62. Hampson M, Driesen NR, Skudlarski P, Gore JC and Constable RT: Brain connectivity related to working memory performance. J Neurosci 26: 13338-13343, 2006

63. Gregory S, Long JD, Tabrizi SJ and Rees G: Measuring compensation in neurodegeneration using MRI. Curr Opin Neurol 30: 380-387, 2017.

64. Spagna A, Kim TH, Wu T and Fan J: Right hemisphere superiority for executive control of attention. Cortex: Dec 29, 2018 (Epub ahead of print). doi: 10.1016/j.cortex.2018.12.012.

65. Chiang S, Stern JM, Engel J Jr, Levin HS and Haneef Z: Differences in graph theory functional connectivity in left and right temporal lobe epilepsy. Epilepsy Res 108: 1770-1781, 2014.

(c) (8) This work is licensed under a Creative Commons Attribution-NonCommercial 4.0 International (CC BY-NC 4.0) License. 